\title{
BMJ Open Quality A quality improvement project aimed at adapting primary care to ensure the delivery of evidence-based psychotherapy for adult anxiety
}

\author{
Mark D Williams, ${ }^{1}$ Craig N Sawchuk, ${ }^{1}$ Nathan D Shippee, ${ }^{2}$ Kristin J Somers, ${ }^{1}$ \\ Summer L Berg, ${ }^{3}$ Jay D Mitchell, ${ }^{4}$ Angela B Mattson, ${ }^{5}$ David J Katzelnick ${ }^{1}$
}

To cite: Williams MD, Sawchuk CN, Shippee ND, et al. A quality improvement project aimed at adapting primary care to ensure the delivery of evidence-based psychotherapy for adult anxiety.BMJ Open Quality 2018;7:e00066. doi:10.1136/ bmjoq-2017-000066

Received 25 March 2017 Revised 20 November 2017 Accepted 21 November 2017

\section{(a) CrossMark}

${ }^{1}$ Department of Psychiatry and Psychology, Mayo Clinic, Rochester, Minnesota, USA ${ }^{2}$ Division of Health Policy and Management, University of Minnesota School of Public Health, Minneapolis, Minnesota, USA

${ }^{3}$ Department of Social Work, Mayo Clinic, Rochester,

Minnesota, USA

${ }^{4}$ Department of Family Medicine, Mayo Clinic, Rochester,

Minnesota, USA

${ }^{5}$ Department of Nursing, Mayo Clinic, Rochester, Minnesota, USA

Correspondence to Dr Mark D Williams; williams.mark@mayo.edu

\section{ABSTRACT}

Primary care patients frequently present with anxiety with prevalence ratios up to $30 \%$. Brief cognitive-behavioural therapy (CBT) has been shown in meta-analytic studies to have a strong effect size in the treatment of anxiety. However, in surveys of anxious primary care patients, nearly $80 \%$ indicated that they had not received CBT. In 2010, a model of CBT (Coordinated Anxiety Learning and Management (CALM)) adapted to primary care for adult anxiety was published based on results of a randomised controlled trial. This project aimed to integrate an adaptation of CALM into one primary care practice, using results from the published research as a benchmark with the secondary intent to spread a successful model to other practices. A quality improvement approach was used to translate the CALM model of CBT for anxiety into one primary care clinic. Plan-Do-Study-Act steps are highlighted as important steps towards our goal of comparing our outcomes with benchmarks from original research. Patients with anxiety as measured by a score of 10 or higher on the Generalized Anxiety Disorder 7 item scale (GAD-7) were offered CBT as delivered by licensed social workers with support by a $\mathrm{PhD}$ psychologist. Outcomes were tracked and entered into an electronic registry, which became a critical tool upon which to adapt and improve our delivery of psychotherapy to our patient population. Challenges and adaptations to the model are discussed. Our 6-month response rates on the GAD-7 were $51 \%$, which was comparable with that of the original research (57\%). Quality improvement methods were critical in discovering which adaptations were needed before spread. Among these, embedding a process of measurement and data entry and ongoing feedback to patients and therapists using this data are critical step towards sustaining and improving the delivery of CBT in primary care.

\section{PROBLEM}

The primary care clinics at Mayo in Rochester, Minnesota, serve over 100000 adult patients at five sites. Access to psychopharmacological support to primary care providers for their adult patients with depression has been available via a care coordination model since 2008 along with direct consultation by psychiatry and advance practice nursing. In 2010, our group began looking for models to increase the availability of on-site cognitive-behavioural therapy (CBT) to our practices. Psychotherapy was available in primary care at some sites but not others via two $\mathrm{PhD}$ psychologists (one part time), one master's level psychologist and one licensed social worker (LICSW) providing primarily supportive therapy. This meant that the majority of primary care patients were referred out of primary care for psychotherapy services. Analysis of practice data found that approximately $50 \%$ of these referred patients either did not schedule or failed to attend an initial psychotherapy consultation session. In our adult care coordination model for depression, we found that comorbid anxiety disorders among depressed patients predicted worse outcomes.

Our group began to look for a model of evidence-based psychotherapy delivery in primary care to adapt to our setting. In 2010, researchers Peter Roy-Bryne, MD, and Michelle Craske, PhD, published evidence from a randomised controlled trial (RCT) on a model of CBT (Coordinated Anxiety Learning and Management (CALM)) adapted to primary care. ${ }^{1}$ As our primary aim for this project, our group set out to use quality improvement (QI) methods to translate this model into one of our clinics using the published randomised trial data as a benchmark to see if we could achieve comparable 6-month response rates (over $50 \%$ response rate at 6 months). A secondary aim was to spread the model to our other sites based on our learnings.

\section{BACKGROUND}

Although primary care has become the 'de facto' mental health system in the USA, significant barriers exist when attempting to connect patients with evidence-based psychotherapy. A survey of over 1000 primary care patients with anxiety found that only $21 \%$ 
had received $\mathrm{CBT}^{2}{ }^{2}$ A 2012 review of over 100 meta-analytic articles concluded that CBT had the strongest degree of impact and efficacy for the treatment of anxiety disorders, ${ }^{3}$ such that, it might be considered a reliable first-line therapy for anxiety.

Anxiety disorders are commonly found in primary care populations with prevalence estimates internationally ranging up to $31 \% .{ }^{4} \mathrm{CBT}$ for anxiety as delivered in primary care settings has shown the most robust results as compared with other short-term modalities. ${ }^{5}$ The primary care setting has advantages (eg, early treatment, reduced stigma) and disadvantages (eg, fast pace, diverse patient population) as a place for incorporating psychotherapy into practice. Efforts at incorporating evidencebased therapy into primary care have included a range of therapy approaches (eg, CBT, problem-solving therapy, supportive therapy) and various modes of delivery (eg, self-help, internet, education of primary care providers in CBT delivery). ${ }^{6}$ When compared with practice as usual and looking at multiple modes of delivery, there is overall support for the incorporation of evidencebased therapy into primary care as a cost-effective addition to services. ${ }^{7}$

CALM $^{1}{ }^{8-12}$ was the largest multisite, randomised controlled CBT trial in primary care for generalised anxiety, social anxiety, panic disorder and post-traumatic stress disorder (PTSD). CALM provided CBT directly within the primary care setting and demonstrated functional and symptomatic improvements in all disorders (excepting PTSD), along with a positive incremental net benefit in quality-adjusted life years.

\section{MEASUREMENT}

In our benchmark for this project, the CALM RCT, ${ }^{1}$ the primary outcome chosen was a change on measures of psychic and somatic anxiety on the 12-item Brief Symptom Inventory (BSI). ${ }^{13}$ Response was defined as a $\geq 50 \%$ reduction on the BSI. Our group had already imbedded an alternative tool into primary care settings. The Generalized Anxiety Disorder 7 (GAD-7) has been widely adopted in primary care settings as a way to identify generalised anxiety disorder. ${ }^{14}$ This tool is strong on ease of use in a busy primary care setting but weak on the ability to distinguish between anxiety disorders (panic disorder, social anxiety disorder, PTSD, etc).

To achieve clarity in diagnosis, the original published research included a structured clinical interview, using the Mini International Neuropsychiatric Interview $(\mathrm{MINI})^{15}$ (approximately $45 \mathrm{~min}$ per patient). For efficiency's sake, our therapists were trained on the use of only those aspects of the MINI specifically targeted to our anxiety disorders of interest (generalised anxiety disorder, panic disorder, social anxiety disorder and PTSD). The primary outcome measure for the project was evaluating all eligible patients (intent to treat) on changes in GAD-7 scores at entry to those at 6 months. Mayo Clinic Institutional Review Board approved this QI project.

\section{DESIGN}

Mayo providers were trained in the CALM model by its originators in late 2010. A multidisciplinary steering group met weekly and included desk staff, nursing, a primary care provider champion, two social workers, a PhD-level psychologist, psychiatry and a QI facilitator.

In the original CALM model, the research therapists included social workers, nurses and psychologists. Our group chose to train the LICSWs as primary therapists for this project. This choice was driven partly by relative costs (as compared with $\mathrm{PhD}$ psychologists), the capacity of LICSW providers to bill independently and their presence already in primary care settings where they provided traditional social work services (eg, finding insurance, nursing homes, etc). The new role of LICSW providers was adapted to become a triple role of traditional social work, evidence-based psychotherapy and a triage function for primary care providers needing on-site input on where to go next with a patient with psychosocial needs. Social work leadership was critical in making this change. We also realised that we needed a way to ensure ongoing fidelity to evidence-based CBT in order to sustain this model, and thus a regular case review process with a $\mathrm{PhD}$ psychology expert was developed. To supplement clinical notes and allow monitoring of fidelity and the ability to learn from our practice, an electronic registry was developed to be used by all therapists in tracking their patient's symptom changes on the GAD-7 and to record what type of intervention was offered at various stages in care.

Patients eligible for the adapted CALM model were adults aged 18 years and older who were diagnosed with at least one of the four previously mentioned anxiety disorders or anxiety disorder not otherwise specified and who agreed to participate. Comorbid depression was not an exclusion to participate. Patients choosing CBT were informed that they would generally be receiving 6-12 sessions in primary care. The final therapy session (described as 'keep going') for treatment completers involved relapse prevention with particular emphasis on the use of exposure techniques to help maintain gains. These patients were considered 'graduates' even if not in remission from a symptomatic perspective. The patient flow is depicted in figure 1 .

\section{STRATEGY}

Implementation of this approach started with a single primary care provider's panel with spread to cover the entire primary care team at the initial site. A qualitative summary of changes in provider attitudes regarding access and availability of treatment resources for anxiety patients in primary care are summarised elsewhere. ${ }^{16}$

\section{Plan-Do-Study-Act cycles}

During the implementation period at the original site, over 25 Plan-Do-Study-Act (PDSA) ${ }^{17}$ cycles were involved in adapting the model to our system. Each PDSA cycle required significant involvement from all members 


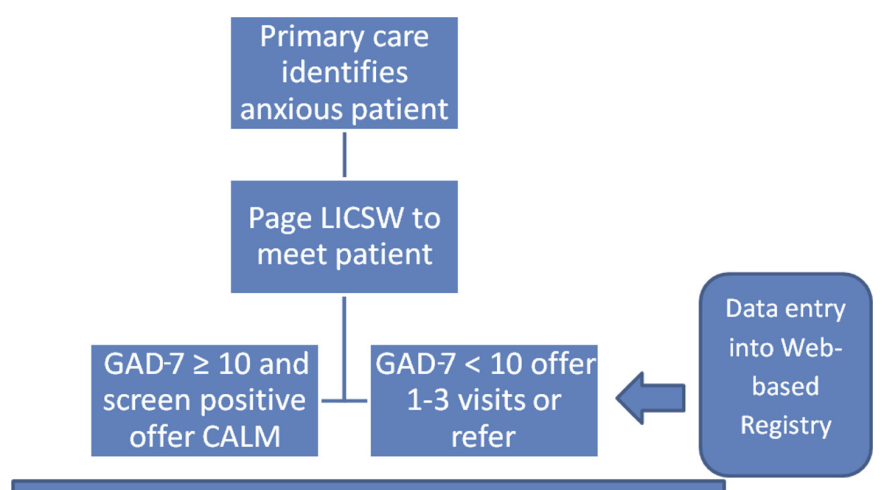

Weekly Case Review with PHD expert

Includes review of data in Registry for missing

data/outcomes

Figure 1 Patient flow. CALM, Coordinated Anxiety Learning and Management; GAD-7, Generalized Anxiety Disorder

7 Item scale; LICSW, licensed social worker.

of our multidisciplinary steering group. Throughout this process, certain workflows were identified as being non-essential or inefficient and therefore were eliminated to further streamline our implementation strategy. Four of the most impactful PDSA cycles are highlighted here, chosen as having the most relevance for clarifying methods for disseminating this model to other primary care locations. The challenges that were addressed in these cycles included $^{1}$ the identified need for an improved process for primary care teams to link the appropriate patient with the appropriate behavioural health provider, ${ }^{2}$ the development of a very brief therapy path to accommodate those referrals with subthreshold symptoms, ${ }^{3}$ a need to ensure adequate documentation of interventions and outcomes by therapy staff and ${ }^{4}$ a decision required on the inclusion of an algorithmic approach to pharmacological treatment of anxiety. These PDSA cycles are summarised in table 1.

\section{RESULTS}

\section{Patient outcomes as compared with benchmark}

To examine the translation and benchmarking of this practice improvement programme against the original CALM protocol and outcomes, we gathered demographic and clinical information on eligible patients and treatment response outcomes for those in an intent-totreat (ITT) group. This group included individuals who entered and completed the programme, dropped out or were administratively inactivated.

Table 1 Plan-Do-Study-Act cycles of change during implementation

\begin{tabular}{|c|c|c|c|c|}
\hline Problem & Plan & Do & Study & Act \\
\hline $\begin{array}{l}\text { Primary care confusion } \\
\text { on how to link patients } \\
\text { with correct behavioural } \\
\text { health provider } \\
\text { as evidenced by } \\
\text { inappropriate referrals. }\end{array}$ & $\begin{array}{l}\text { Reorganise ordering } \\
\text { from type of provider } \\
\text { (PhD, LICSW, } \\
\text { MD) to type of } \\
\text { service (diagnostic } \\
\text { evaluation, medication } \\
\text { management, therapy). }\end{array}$ & $\begin{array}{l}\text { Rollout of new } \\
\text { electronic ordering. }\end{array}$ & $\begin{array}{l}\text { Frequency of } \\
\text { inappropriate referrals } \\
\text { went down overall } \\
\text { except for those from a } \\
\text { few individual providers. }\end{array}$ & $\begin{array}{l}\text { One-on-one assistance } \\
\text { on the new ordering } \\
\text { system offered to those } \\
\text { slow to adapt to new } \\
\text { system. }\end{array}$ \\
\hline $\begin{array}{l}\text { Reference RCT CALM } \\
\text { model also included an } \\
\text { algorithm for medication } \\
\text { management of anxiety. }\end{array}$ & $\begin{array}{l}\text { Offer medication review } \\
\text { for patients with anxiety } \\
\text { as a part of parallel } \\
\text { adult care coordination } \\
\text { available in primary care } \\
\text { settings. }\end{array}$ & $\begin{array}{l}\text { Algorithm created } \\
\text { and reviewed, option } \\
\text { for medication review } \\
\text { advertised. }\end{array}$ & $\begin{array}{l}\text { Referral rates by primary } \\
\text { care very low for routine } \\
\text { review of medications. }\end{array}$ & $\begin{array}{l}\text { Abandoned routine } \\
\text { algorithm for anxiety } \\
\text { medications in favour of } \\
\text { individual consultation } \\
\text { as needed. }\end{array}$ \\
\hline
\end{tabular}

CALM, Coordinated Anxiety Learning and Management; GAD-7, Generalized Anxiety Disorder 7 Item scale; LICSW, licensed social worker; $\mathrm{RCT}$, randomised controlled trial. 
Table 2 Comparing demographics with published CALM results

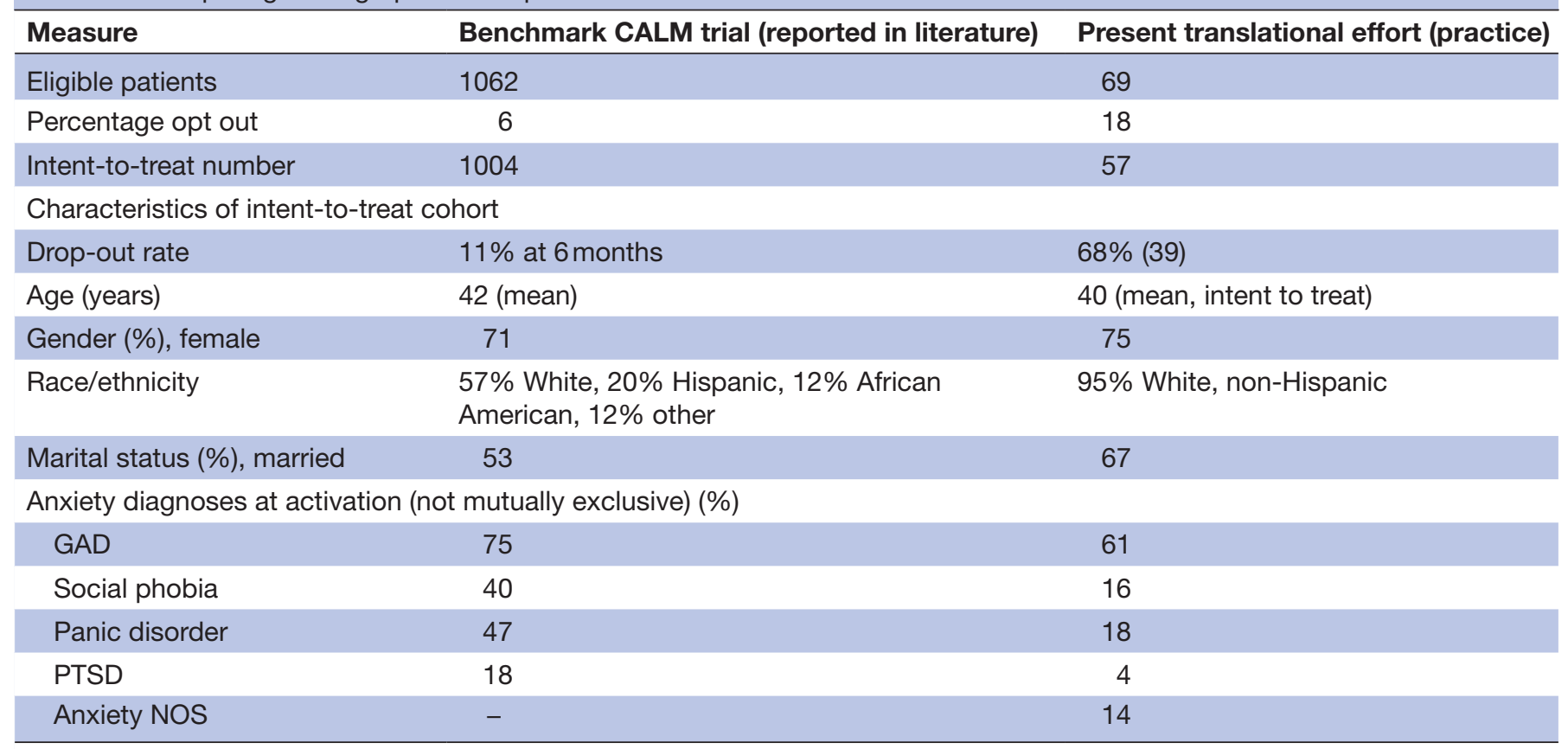

CALM, Coordinated Anxiety Learning and Management; GAD, generalised anxiety disorder; NOS, not otherwise specified; PTSD, posttraumatic stress disorder.

\section{Data analysis}

Statistical analyses were conducted using StataMP V.12.1 (StataCorp LP, Stata/MP 12.1 for Windows, 2011). Due to the modest sample size for these initial findings, inferential statistics and modelling were limited. Analyses and results described here are limited to those individuals who scored a 10 or higher on the baseline GAD- $7^{14}$ between August 2011 and August 2014. Additional exclusion criteria for evaluating effectiveness included not having an identified anxiety diagnosis at enrolment or activation and those who did not agree to participate ('opt-outs').

We first employed descriptive analyses of baseline and follow-up information on demographics, GAD-7 and clinical and treatment information, as well as information on rates of opting out and numbers in the ITT cohort. We also compared our findings with the original CALM outcomes published in the empirical literature. ${ }^{1}$ Next, we assessed treatment response rates (defined as $50 \%$ or greater reduction on the GAD-7) based on that last GAD-7 score carried forward and compared them with published CALM trial response rates. Finally, we looked at a completer group defined as those who reached the final 'keep going' session versus all others on rates of GAD-7 response at 6 months.

\section{Demographic and clinical baseline data in our population versus published model}

Basic demographic and clinical characteristics at baseline are shown in table 2, comparing the characteristics of the CALM trial (research) with the present practice cohort. Based on an overall sample of $n=69$ and an ITT cohort of $n=57$, the practice-based implementation of CALM had larger percentages of patients opt-out, drop-out or non-complete. The practice-based implementation's ITT cohort was also slightly younger and had greater proportions of females, White non-Hispanics and married individuals in comparison with the research ITT group. In terms of diagnoses at activation into CALM, the present practice-based implementation had lower rates of the four main anxiety diagnoses used in the research trial. This finding was possibly based on less attention to comorbid anxiety diagnoses and more patients with non-specific anxiety diagnoses being referred into the programme.

\section{Treatment response and comparison with previous trial}

Treatment response at 6 months as a function of GAD-7 change scores is shown in table 3 for individuals in the ITT group, as compared with the research trial. Treatment response was $50.9 \%$ on the GAD-7, in the practice-based setting as compared with $57 \%$ in the trial. Of the ITT cohort $(n=57), 28.1 \%(n=16)$ completed CALM by reaching the 'keep going' session; the remaining patients $(n=41)$ may have improved but for multiple

Table 36 months treatment response and comparison with benchmark Coordinated Anxiety Learning and Management trial

Translational

Measure Benchmark BSI-12 effort GAD-7

$\begin{array}{llll}\text { Treatment } & 95 \% \mathrm{Cl} & 95 \% \mathrm{Cl} \\ \text { response rate } & 57 \% 52.8 \text { to } 62.1 & 51 \% 37.5 \text { to } 64.3 \\ \begin{array}{l}(\geq 50 \% \text { reduction } \\ \text { in symptoms) }\end{array} & & & \\ \end{array}$

BSI-12, Brief Symptom Inventory 12; GAD-7, Generalized Anxiety Disorder 7. 
reasons did not complete the final session as designed in the intervention. Among completers, 93.8\% (all but 1) had a treatment response, compared with a treatment response rate of just $34.2 \%$ among non-completers. A $\chi^{2}$ test of association was significant $(\mathrm{P}<0.001)$.

The mean number of treatment sessions by the 6 -month evaluation period for all patients was 4.7. Those with the highest symptom ratings on the GAD-7 at baseline (scores of 16-21) had statistically fewer sessions and the lowest treatment response rate, with a higher tendency than the lower baseline-symptom groups to drop out of care.

\section{LESSONS AND LIMITATIONS}

From a practice perspective, the implementation of CALM as a vehicle for incorporating CBT into real-world primary care settings was a success, resulting in dissemination to other sites. Primary care providers appreciated clarity on how to gain assistance for their patients and were more satisfied with improved access. ${ }^{16}$ Clinical outcomes included over $50 \%$ response at 6 months on the primary anxiety tool (GAD-7), which is comparable with the original study $(57 \%)$, but this observational review lacked resources to create a prospective control group within the practice for better comparison.

Some of the choices made in this process are common adaptations when translating treatments from bench to bedside (eg, who will provide therapy, how to do training, which outcome measures to use and how to track outcomes). Some of the choices may have led to a 'voltage drop' in outcomes (eg, less restrictive patient population, lack of dedicated resources to catch missing data).

We also learnt important lessons about adaptations in primary care to maintain fidelity to evidence-based treatment. CBT is based on learning cognitive and behavioural approaches to specific problems and routinely tracking outcomes. To track these outcomes on a population of patients, we found that we needed to routinely enter data into a registry-a new task for psychotherapists in our system. Registries often serve more than one function, and in our case, the registry allows the opportunity to evaluate patient progress, fidelity to $\mathrm{CBT}$ and areas for programme development.

This project reports on our ability to address anxiety in primary care. Patients in primary care have a multitude of therapy needs, including crisis support, treatment of conditions other than anxiety, assistance in addressing relationship challenges, therapy for personality disorders, and so on. The model described was broadened to manage depression as well as anxiety; however, an ongoing challenge has been to find ways to link to community resources for those aspects of psychotherapy (eg, dialectic behavioural therapy for borderline personality disorder), which might overwhelm our resources and diminish access.

Issues of bias and internal validity exist due to the non-controlled, selective sample and routine practice setting. While addressing practical questions in a real-world setting, this study provides evidence of limited strength regarding the effectiveness of CALM. Nonetheless, this work remains important due to the gap between evidence supporting the effectiveness of adapted CBT for anxiety in primary care and the lack of reliable access to this service for our patients in primary care settings.

\section{CONCLUSION}

While CBT has been tested in primary care settings as described earlier, there is very little published to guide practices on how to take such models and make them happen in real-world settings. Through modification of CALM and adaptations to our practice, we were successful at obtaining results that were comparable with our benchmark, which was the published outcomes from original effectiveness study. ${ }^{1}$ One major advantage of the approach presented in this research is the incorporation into practice of a method of evidence gathering while providing the treatment. A common challenge to the implementation of any therapy programme is the risk of 'drift' away from applying an evidence-based therapy approach to a given mental health problem once the research or oversight is done.$^{18}$ Fidelity requires data and monitoring. Our programme implemented and normalised the need for therapists to do their own data entry on a measurable outcome into a registry with regular meetings to review what modalities of therapy were applied to which patient groups, allowing ongoing attention to individual therapists who need extra support, and the capacity to add new indications (eg, insomnia) and assess outcomes. We have sustained this process, for many reasons including the fact that feedback using measures to patients and providers in and of itself has been shown to lead to better outcomes. ${ }^{19}$

In regard to next steps, this model was well supported and has spread to all five clinics in our primary care system in Rochester, Minnesota. We have added depression (measured with the Patient Health Questionnaire-9) and are adding insomnia to our indications for therapy. As we explore future spread to our network of primary care clinics in rural areas and other states, we have several challenges to overcome. These include ensuring data entry and the use of a registry across geographical areas, local regulations on therapy, adapting the model to be available via telemedicine options and attempting to keep the model of therapy brief in settings where alternative therapy resources are less available. We value the ability to have meaningful feedback on progress to patients and providers, which requires ongoing attention to finding measures that balance the need for meaningful measurement and the requirement for tools that are brief and simple for this setting.

Acknowledgements We would like to acknowledge the assistance and support provided during this project by Amy Glasgow of the Robert D and Patricia E Kern Center for the Science of Health Care Delivery. This work was supported by the Robert D and Patricia E Kern Center for the Science of Health Care Delivery.

Contributors Design and implementation of the quality improvement effort: MW, CNS, KJS, SLB, JDM, ABM and DJK. Plan study: MW, NDS, ABM and DJK. Data acquisition, cleaning, analysis and review: MW, CNS, NDS, ABM and DJK. 
Manuscript preparation, review and editing: MW drafted the initial manuscript and all authors contributed to editing and review. Submitting the study: MW.

Competing interests MW has consulted with the University of Washington, AIMS Center. MDW has also done peer review for the Neuroscience Education Institute NEI. DJK is a principal of Healthcare Technology Systems Inc. DJK has consulted for the National Network of Depression Centers. NDS, KJS, SLB, JDM, and ABM have identified no potential conflicts of interest.

Provenance and peer review Not commissioned; externally peer reviewed.

Open Access This is an Open Access article distributed in accordance with the Creative Commons Attribution Non Commercial (CC BY-NC 4.0) license, which permits others to distribute, remix, adapt, build upon this work non-commercially, and license their derivative works on different terms, provided the original work is properly cited and the use is non-commercial. See: http://creativecommons.org/ licenses/by-nc/4.0/

(c) Published by the BMJ Publishing Group Limited. For permission to use (where not already granted under a licence) please go to http://www.bmj.com/company/ products-services/rights-and-licensing/

\section{REFERENCES}

1. Roy-Byrne P, Craske MG, Sullivan G, et al. Delivery of evidencebased treatment for multiple anxiety disorders in primary care: a randomized controlled trial. JAMA 2010;303:1921-8.

2. Stein MB, Roy-Byrne PP, Craske MG, et al. Quality of and patient satisfaction with primary health care for anxiety disorders. J Clin Psychiatry 2011;72:970-6.

3. Hofmann SG, Asnaani A, Vonk IJ, et al. The efficacy of cognitive behavioral therapy: a review of meta-analyses. Cognit Ther Res 2012;36:427-40.

4. Kessler RC, Angermeyer M, Anthony JC, et al. Lifetime prevalence and age-of-onset distributions of mental disorders in the World Health Organization's World Mental Health Survey Initiative. World Psychiatry 2007;6:168-76.

5. Cape J, Whittington C, Buszewicz M, et al. Brief psychological therapies for anxiety and depression in primary care: meta-analysis and meta-regression. BMC Med 2010;8:38.

6. Dorflinger LM, AHt F, Foran-Tuller KA. Training primary care physicians in cognitive behavioral therapy: a review of the literature. Patient education and counseling. Review 2016;99:1285-92.
7. Twomey C, O'Reilly G, Byrne M. Effectiveness of cognitive behavioural therapy for anxiety and depression in primary care: a meta-analysis. Fam Pract 2015;32:3-15.

8. Craske MG, Rose RD, Lang A, et al. Computer-assisted delivery of cognitive behavioral therapy for anxiety disorders in primary-care settings. Depress Anxiety 2009;26:235-42.

9. Craske MG, Stein MB, Sullivan G, et al. Disorder-specific impact of coordinated anxiety learning and management treatment for anxiety disorders in primary care. Arch Gen Psychiatry 2011;68:378-88.

10. Sullivan G, Craske MG, Sherbourne C, et al. Design of the coordinated anxiety learning and management (calm) study: innovations in collaborative care for anxiety disorders. Gen Hosp Psychiatry 2007;29:379-87.

11. Rose RD, Lang AJ, Welch SS, et al. Training primary care staff to deliver a computer-assisted cognitive-behavioral therapy program for anxiety disorders. Gen Hosp Psychiatry 2011;33:336-42.

12. Curran GM, Sullivan G, Mendel P, et al. Implementation of the CALM intervention for anxiety disorders: a qualitative study. Implement Sci 2012;7:1-11.

13. Derogatis LR, Melisaratos $N$. The brief symptom inventory: an introductory report. Psychol Med 1983;13:595-605.

14. Spitzer RL, Kroenke K, Williams JB, et al. A brief measure for assessing generalized anxiety disorder: the GAD-7. Arch Intern Med 2006;166:1092-7.

15. Sheehan DV, Lecrubier Y, Sheehan KH, et al. The Mini-International Neuropsychiatric Interview (M.I.N.I.): the development and validation of a structured diagnostic psychiatric interview for DSM-IV and ICD-10. J Clin Psychiatry 1998;59 Suppl 20(Suppl 20):22-33.

16 Vickers KS, Ridgeway JL, Hathaway JC, et al. Integration of mental health resources in a primary care setting leads to increased provider satisfaction and patient access. Gen Hosp Psychiatry 2013;35:461-7.

17 Berwick DM. Developing and testing changes in delivery of care. Ann Intern Med 1998;128:651-6.

18. Waller G, Stringer H, Meyer $C$. What cognitive behavioral techniques do therapists report using when delivering cognitive behavioral therapy for the eating disorders? J Consult Clin Psychol 2012;80:171-5.

19. Gondek D, Edbrooke-Childs J, Fink E, et al. Feedback from outcome measures and treatment effectiveness, treatment efficiency, and collaborative practice: a systematic review. Adm Policy Ment Health 2016;43:325-43. 